Article

\title{
Insect-Mimetic Imaging System Based on a Microlens Array Fabricated by a Patterned-Layer Integrating Soft Lithography Process
}

\author{
Minwon Seo ${ }^{1}$, Jong-Mo Seo ${ }^{1,2, *}$, Dong-il “Dan" Cho ${ }^{1}$ and Kyo-in Koo ${ }^{3, *}$ (]) \\ 1 Department of Electrical and Computer Engineering, Seoul National University, Seoul 08826, Korea; \\ sm7bmw@snu.ac.kr (M.S.); dicho@snu.ac.kr (D.C.) \\ 2 Biomedical Research Institute, Seoul National University Hospital, Seoul 03080, Korea \\ 3 Department of Biomedical Engineering, University of Ulsan, Ulsan 44610, Korea \\ * Correspondences: callme@snu.ac.kr (J.-M.S.); kikoo@ulsan.ac.kr (K.K.); Tel.: +82-2-880-1739 (J.-M.S.)
}

Received: 16 May 2018; Accepted: 21 June 2018; Published: 22 June 2018

\begin{abstract}
In nature, arthropods have evolved to utilize a multiaperture vision system with a micro-optical structure which has advantages, such as compact size and wide-angle view, compared to that of a single-aperture vision system. In this paper, we present a multiaperture imaging system using a microlens array fabricated by a patterned-layer integrating soft lithography (PLISL) process which is based on a molding technique that can transfer three-dimensional structures and a gold screening layer simultaneously. The imaging system consists of a microlens array, a lens-adjusting jig, and a conventional (charge-coupled device) CCD image sensor. The microlens array has a light screening layer patterned among all the microlenses by the PLISL process to prevent light interference. The three-dimensionally printed jig adjusts the microlens array on the conventional CCD sensor for the focused image. The manufactured imaging system has a thin optic system and a large field-of-view of 100 degrees. The developed imaging system takes multiple images at once. To show its possible applications, multiple depth plane images were reconstructed based on the taken subimages with a single shot.
\end{abstract}

Keywords: microlens array; insect-mimetic; multiaperture vision system

\section{Introduction}

Most conventional imaging systems adopt a single aperture structure inspired by vertebrates' vision system. Stacking lenses along the optical axis enables this imaging system to achieve a wide-angle view, a telescopic view, and reduced aberrations. However, the single-aperture vision system has disadvantages, such as the limitation of miniaturization and low time resolution [1]. To overcome these disadvantages, efforts have been made to mimic insects' vision system.

Figure 1 shows a schematic illustration of anatomical structures of the insect compound eye. There are two types of compound eye: an apposition compound eye and a superposition compound eye [2]. The apposition compound eye receives light through hundreds to thousands of facets. Every facet has a pigment to prevent light from entering neighborhood facets. This enables a distinct point pixel instead of a cross-talked white Gaussian noised pixel [3,4]. Oppositely, insects with a superposition compound eye, which are those insects that usually move around at nighttime, do not have the pigment unit among the facets. This pigmentless structure causes light interference on photoreceptors and blurs object images. However, superposed light in the pigmentless facets enables the nocturnal insect to react more sensitively to the nighttime environment $[5,6]$. 


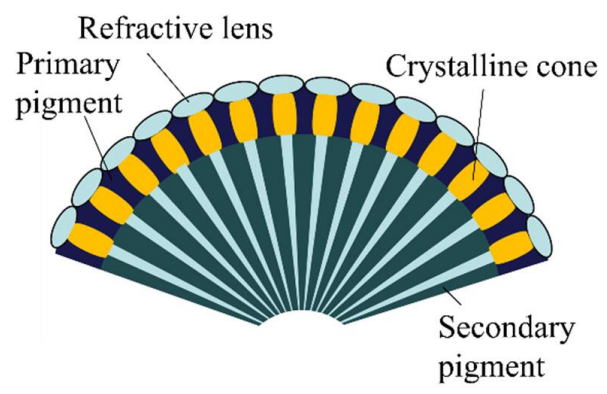

(a)

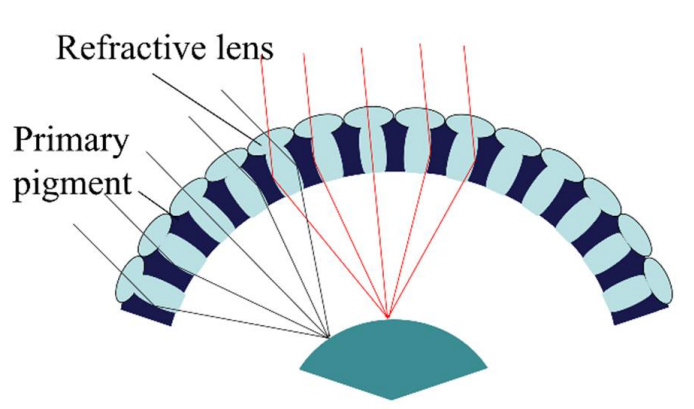

(b)

Figure 1. The schematic diagram of (a) the apposition compound eye and (b) the superposition compound eye.

To mimic the structure of the compound eye, a microlens array has been widely used [7-10]. To fabricate the microlens array, a thermal reflow process is usually employed because of its reliable spherical profile as well as its simple fabrication process.

There are several works mimicking the compound eye of Xenos peckii, which envisions subimages of the object with its clustered eyes [11-13]. To perceive high-quality subimages from the compound eye, a light screening layer among the microlenses is indispensable. Morgan et al. [14] implemented an opaque wall to change the color of glasses among microlenses using thermal treatment. Ichioka et al. [15] inserted optical separators between an image sensor and a microlens array. This method required aligning process of the optical separator to the microlenses. Kim et al. [16] made a perforated black polymer sheet and covered a curved microlens array with the black sheet to block the interference of light. The abovementioned techniques used to achieve a microlens array with a screening layer require manual alignment or high thermal treatment.

In this paper, we presented a compound eye mimicking an imaging system using a microlens array integrated with a gold screening layer fabricated by a patterned-layer integrating soft lithography (PLISL) process. The microlens array integrated with the light screening pattern was adjusted on a conventional charge-coupled device (CCD) image sensor by a lens-adjusting jig. The captured image was refocused in three different planes to demonstrate depth perception capability. Moreover, in order to demonstrate the extensibility of our PLISL process, a microlens array with a gold screening layer was fabricated on a spherical surface as well.

\section{Materials and Methods}

\subsection{Fabrication of a Microlens Array with a Gold Screening Layer}

In order to determine the focal length of the microlens, the thickness of the glass protecting the image sensor should be considered. The thickness of the protecting glass, as measured by a caliper, was $0.44 \mathrm{~mm}$. It was assumed that the glass has a refractive index similar to N-BK7 glass, which has a refractive index of 1.517. Therefore, the focal length of the microlens should be above $0.67 \mathrm{~mm}$.

Since the thermal reflow process enables a cylindrical photoresist structure to transform into a microlens structure due to the property of minimizing surface energy [17], the height $(\mathrm{H})$ and diameter (2r) of the cylindrical structure decides the curvature of the microlens. The height of a cylinder with $10 \mu \mathrm{m}$ and a refractive index of UV curing resin is 1.56 [18]. Therefore, the height of the microlens can be calculated by Equations (1) and (2). The radius of the microlens should be above $118 \mu \mathrm{m}$. In this paper, the radius of the microlens array was set to $175 \mu \mathrm{m}$ for the thin optical system and stable focus adjustability (See Supplementary Information).

$$
\pi r^{2} H=\frac{\pi h}{6}\left(3 r^{2}+h^{2}\right)
$$




$$
\begin{gathered}
\mathrm{h}=\sqrt[3]{\mathrm{X}}-\frac{r^{2}}{\sqrt[3]{X}} \text { for } \mathrm{X}=\sqrt{9 H^{2} r^{4}+r^{6}}+3 H r^{2} \\
\mathrm{f}=\frac{r^{2}+h^{2}}{2 h(n-1)}
\end{gathered}
$$

The soft lithography process is widely used to fabricate three-dimensional microstructures. Even though the soft lithography process has several advantages, including repeatability and simplicity, it is not easy to add another patterned layer on the surface of the soft-lithography-processed polymer. In this investigation, to fabricate a screening layer among the microlenses, a PLISL process was proposed, as shown in Figure 2. Basically, the proposed process was an advanced version of the soft lithography process. A gold film which has weak adhesion to a silicon wafer [19] was used as a light screening material. It was patterned on a silicon substrate without any adhesion-promoting layer like titanium or chrome. On the gold prepatterned substrate, a three-dimensional microstructure was implemented and transferred to the polymer material by molding. During the molding process, the prepatterned gold film was transferred and integrated onto the polymer because of its low adhesion to the silicon wafer.

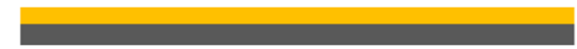

(a)

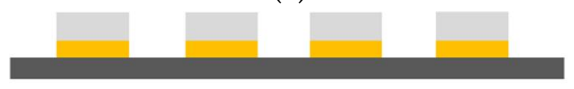

(c)

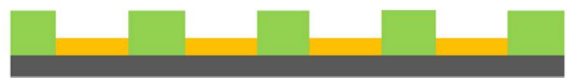

(e)

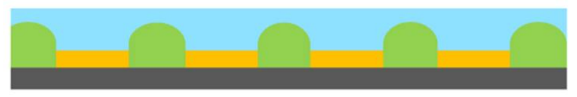

(g)

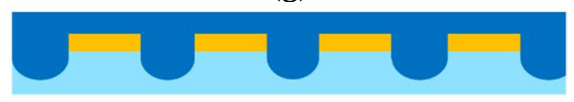

(i)

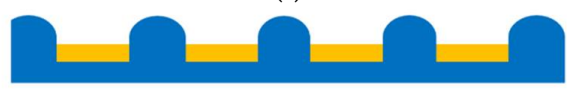

(k)

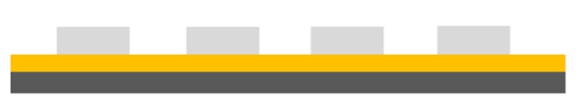

(b)

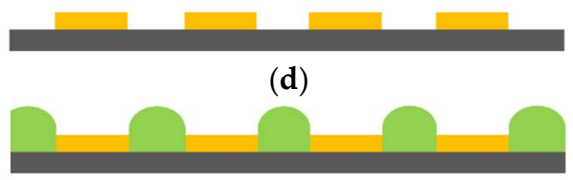

(f)

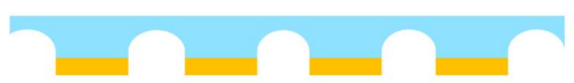

(h)

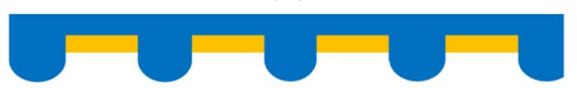

(j)

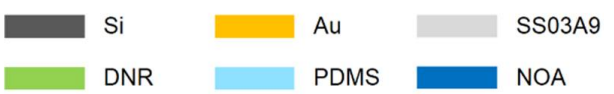

Figure 2. The specific steps of the PLISL process. (a) The gold layer was deposited on the silicon; (b) positive PR patterning; (c) gold layer etching; (d) PR removal; (e) negative PR patterning; (f) thermal reflow; (g) 1st molding with the elastomer; (h) the 1st molded layer; (i) 2nd molding with the UV resin; (j) the 2nd molded layer; (k) the fabricated microlens array with gold layer.

The specific steps of the PLISL process are as follows: A silicon wafer was cleaned in a mixture of $\mathrm{H}_{2} \mathrm{SO}_{4}: \mathrm{H}_{2} \mathrm{O}_{2}$ (3:1) for $30 \mathrm{~min}$ at 60 degrees Celsius. After that, a 120-nm thick gold layer [20], which would serve as the light interference screening layer, was deposited on the cleaned wafer by an E-gun evaporator (ZZS550-2/D, Maestech Co., Ltd., Pyungtaek, Korea) without any adhesion layer like titanium or chrome (Figure 2a). A positive photoresist, SS-03A9 (Dongwoo Fine-chem Co., Ltd., Gyeonggi-do, Korea), was patterned on the gold evaporated silicon wafer (Figure 2b). The photoresist patterned wafer was etched by gold etchant, which was a mixture of $\mathrm{I}_{2}: \mathrm{KI}_{2} \mathrm{H}_{2} \mathrm{O}$ (1:4:10) (Figure 2c). After removing the photoresist on the wafer with acetone, the gold-patterned wafer was coated by negative photoresist, DNR (Dongjin Semichem Co., Ltd., Incheon, Korea), about $10 \mu \mathrm{m}$ by a spin coater and baked on a hot plate at 95 degrees Celsius. The second photoresist layer was coated on the first photoresist layer and baked on the hot plate at 110 degrees Celsius. The wafer was exposed using the identical mask with the gold-layer patterning mask to pattern on the gold-free area (Figure 2e). 
After the UV exposed wafer was developed, the developed wafer was baked on a hot plate to melt the photoresist pattern for the spherical structure by the surface tension (Figure 2f). This heating step is widely known as the thermal reflow process.

After the silicon substrate steps, the microlens array pattern and the gold screening layer was transferred using polydimethylsiloxane (PDMS, Sylgard 184, Dow Corning, Scottsdale, AZ, USA) (Figure 2h). To get a convex microlens array with a screening layer, a UV-curable epoxy resin (NOA 72, Norland Products Inc., Cranbury, NJ, USA) with a refractive index 1.56 was poured on the concave-patterned PDMS mold. A transparent polyethylene-terephthalate (PET) film was placed on the poured epoxy resin for flattening and easy handling. The resin was cured by $15 \mathrm{~W}$ UV light with a wavelength of $365 \mathrm{~nm}$ (Figure 2i). The cured and stripped resin had a convex microlens array integrated with the gold layer, as shown in Figure 2k. Finally, a thin parylene was deposited on the convex microlens array and the gold screening layer for electrical isolation from the CCD sensor.

\subsection{Imaging System Setup Using a 3D Printed Jig}

Figure 3 shows a schematic of the proposed imaging system. It consists of a microlens array, a focus-adjustable jig, and a 6.72-mm CCD sensor $(1920 \times 1080$ pixels, P5756, Hanjindata, Gyeonggi-do, Korea). The jig was designed and three-dimensionally printed for secure positioning and focusing of the microlens array. The aperture size of the jig was $4 \mathrm{~mm}$. Screws and springs were used to adjust a four-point focal distance between the microlens array and the CCD sensor.

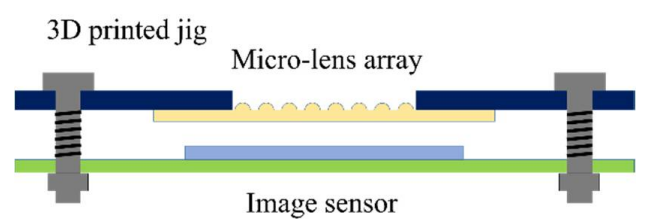

(a)

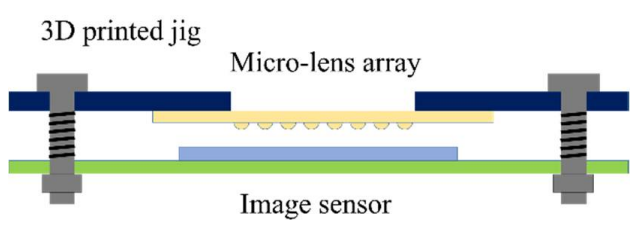

(b)

Figure 3. The schematic diagram of the imaging system setup. (a) The convex part of the microlens array looks outside. (b) The convex part of the microlens array faces the CCD sensor.

There are two possible directions when integrating the microlens array with the sensor. One is that a convex part of the microlens array looks outside (Figure 3a). The other is that the convex part of the microlens array faces the CCD sensor (Figure 3b). In the case of the microlens array facing the CCD sensor, the gold screening layer is closer to the CCD sensor compared with the case of the microlens array looking outside. This closer distance is expected to reduce the interference of the entered light and to enhance imaging quality.

\section{Results}

\subsection{Characteristics of the Microlens Array}

A cylindrical photoresist structure with a height of $10 \mu \mathrm{m}$ and a diameter of $350 \mu \mathrm{m}$ was thermal-reflowed. The microlens mold with a curvature of $778.9 \mu \mathrm{m}\left(\mathrm{r}_{\mathrm{sph}}\right.$ was $175 \mu \mathrm{m}$ and $\mathrm{h}_{\mathrm{sph}}$ was $19.91 \mu \mathrm{m}$ ) was fabricated, as shown in Figure 4a. The focal length of the microlens implemented using this thermal-reflowed mold was $1.442 \mathrm{~mm}$. Spacing among every microlens was fabricated as $500 \mu \mathrm{m}$.

Figure $4 \mathrm{~b}$ shows the concave microlens array and the gold layer that was molded in PDMS on a cover slip glass substrate. By using the UV-imprinting process, the concave pattern was molded in NOA 72 on PET film (Figure 4c).

One of the advantages of our PLISL process is that three-dimensional microstructure and integrated layer is transferred not only on a planar substrate but also on a hemispherical substrate using the widely known negative pressure technique (Figure 5a). Due to the negative pressure, the flexible 
PDMS mold is deformed as the hemispherical structure. Figure $5 b$ shows a microlens array on the hemispherical substrate using a three-dimensionally printed chamber.

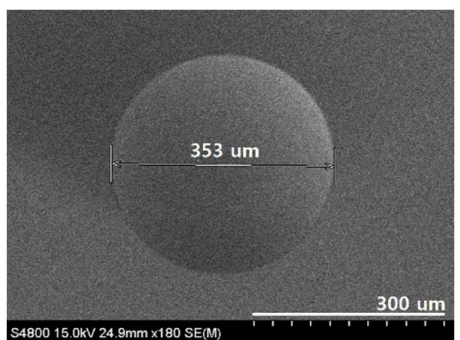

(a)

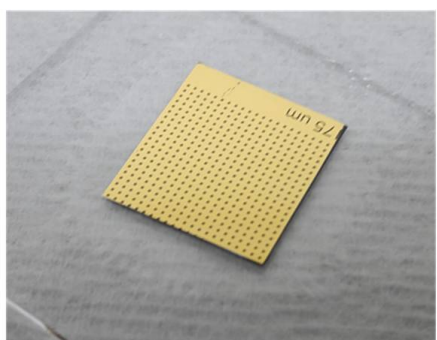

(b)

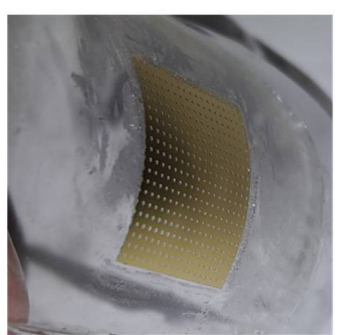

(c)

Figure 4. The fabricated microlens array (a) implemented using thermal reflow process, (b) firstly molded with PDMS on glass substrate and (c) secondly molded with UV curing resin on PET film.

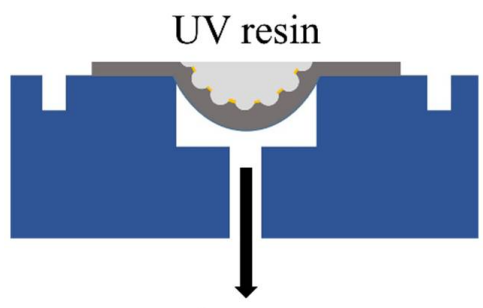

Negative pressure

(a)

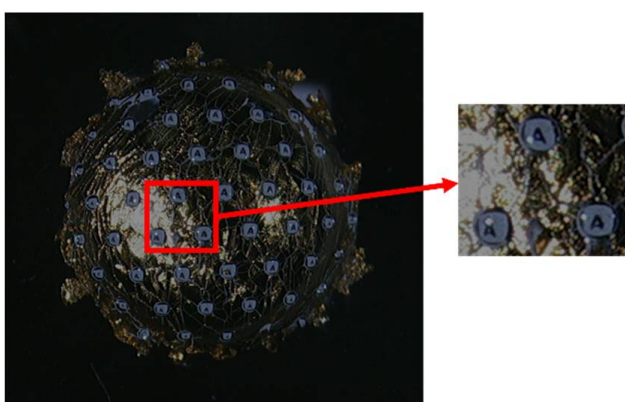

(b)

Figure 5. (a) The fabrication schematic for the hemispherical substrate. (b) The fabricated microlens array with the screening layer on the hemispherical substrate.

\subsection{Integrated Imaging System with a Conventional CCD Image Sensor}

Figure 6 shows the assembled imaging system with the microlens array adjusted by the 3D printed jig, springs, and bolts. Due to the springs and the bolts, the distance between the microlens array and the CCD sensor was calibrated to focus every microlens' image. The light-entering window of the 3D printed jig is a square shape of $4 \times 4 \mathrm{~mm}$. Eighteen microlenses receive light from the light-entering window. The photo sensing part of the CCD sensor is a rectangular shape that is $5.37 \times 4.04 \mathrm{~mm}$ and $1920 \times 1080$ pixels.

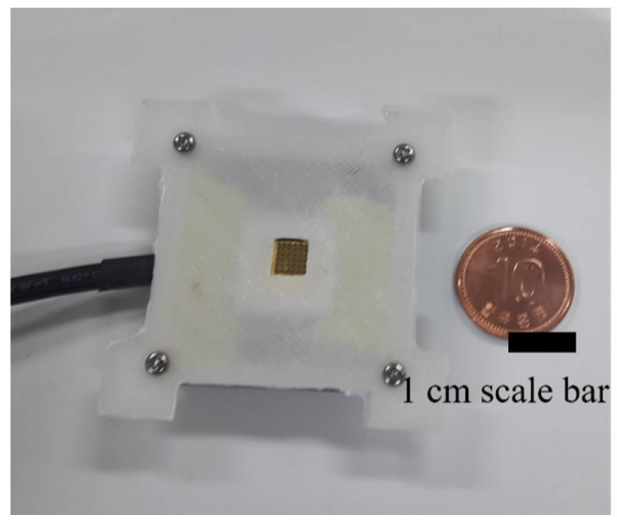

(a)

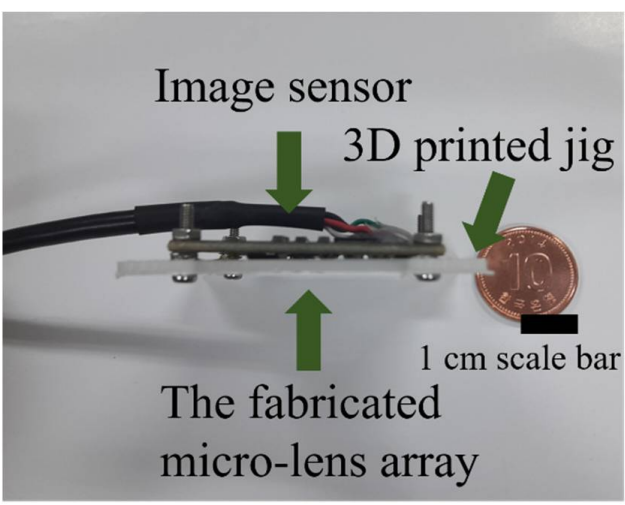

(b)

Figure 6. (a) The fabrication schematic for the hemispherical substrate. (b) The fabricated microlens array with the screening layer on the hemispherical substrate. 
Figure 7 presents images of a printed USAF 1951 pattern captured by two different setups: the microlens-facing-outside setup (Figure 7a) and the microlens-facing-inside setup (Figure 7b). The facing-inside setup shows better contrast around the rim than the looking-outside setup. We could assume that the apertures of the facing-inside setup play a better role because the gold screening layer of the facing-inside setup is closer to the image sensor by a thickness of the NOA substrate.

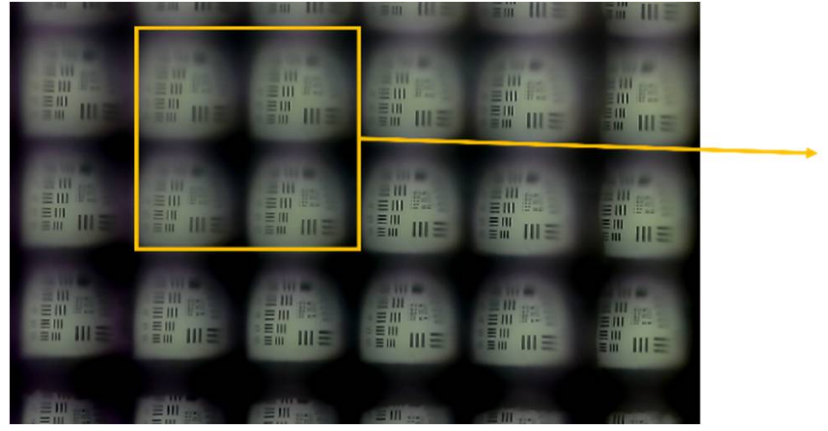

(a)

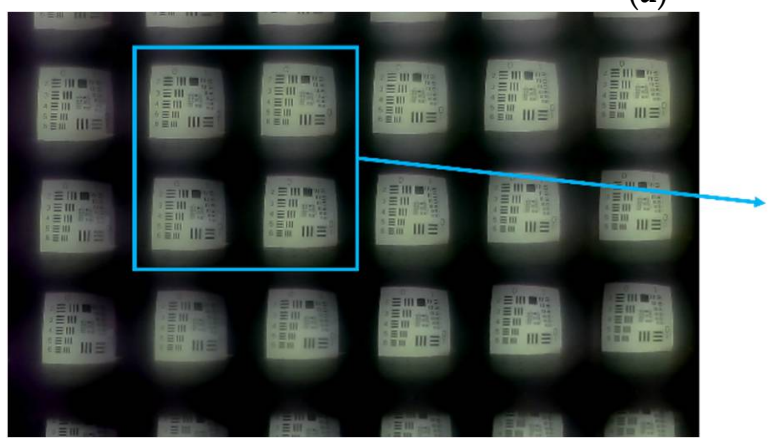

(b)
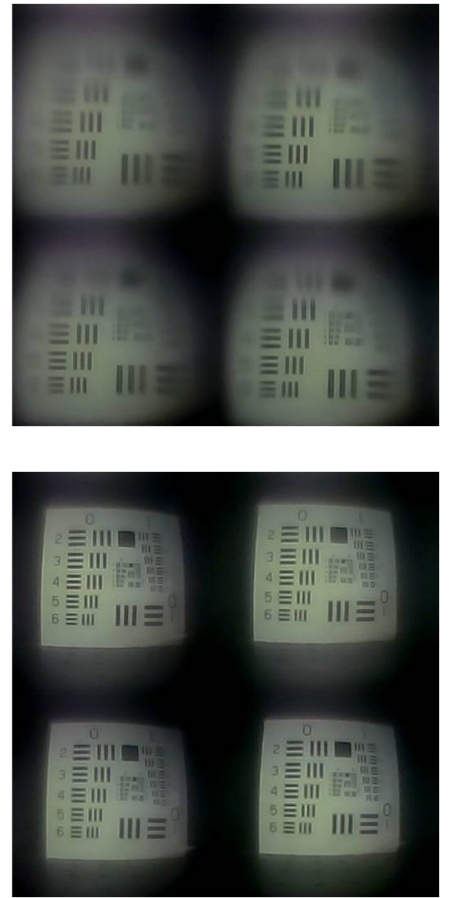

Figure 7. The captured images of the printed USAF 1951 test pattern at a distance of $10 \mathrm{~cm}$ by our imaging system with (a) the microlens-facing-outside setup and (b) the microlens-facing-inside setup.

Field-of-view (FOV) of the assembled imaging system was measured using a 3D-printed half-circle structure and a paper-printed goniometer, as shown in Figure 8a. Figure 8 shows that the leftmost microlens captured -50 degrees and the rightmost microlens took +50 degrees. This means that our imaging system has about 100 degrees of FOV, which is higher than the FOV of the single microlens (80 degrees).

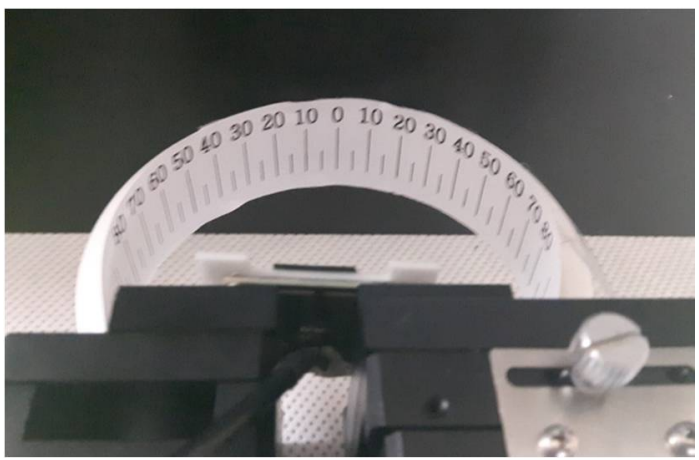

(a)

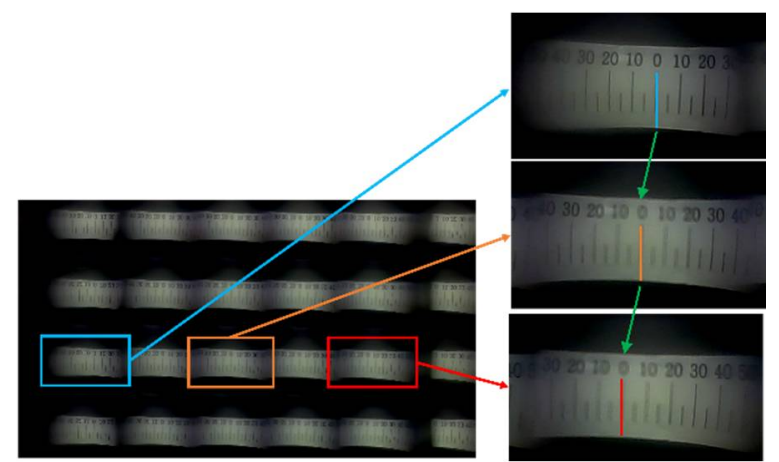

(b)

Figure 8. The experimental setup for measuring the FOV of the assembled imaging system. (a) The captured image represents about 100 degrees of the FOV. (b) The captured images of (upper) the leftmost microlens, (middle) the middle microlens, and (lower) the rightmost microlens. 
Every subimage represents different perspective views of the identical object. Due to this plentiful information, post-image processing of the multiaperture imaging system generates diverse images including higher-resolution images [21] and multifocusing images [22]. Figure 9 shows multifocusing images. Using the 18 subimages of one single shot (Figure 9a), three differently focused images were reconstructed, as shown in Figure $9 \mathrm{~b}-\mathrm{d}$. The characters "SNU", "UoU", and "EFE LAB" are apart $1 \mathrm{~cm}$, $2 \mathrm{~cm}$, and $3 \mathrm{~cm}$ from the imaging system, respectively.

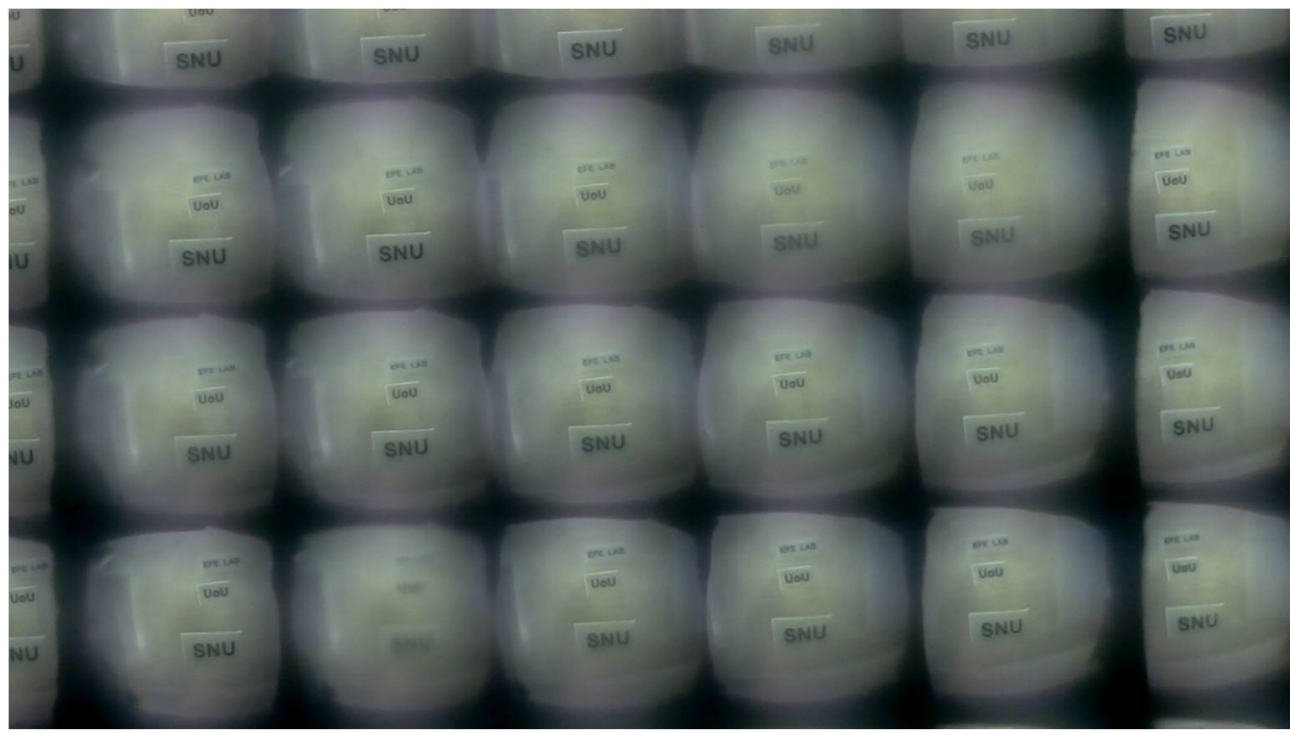

(a)

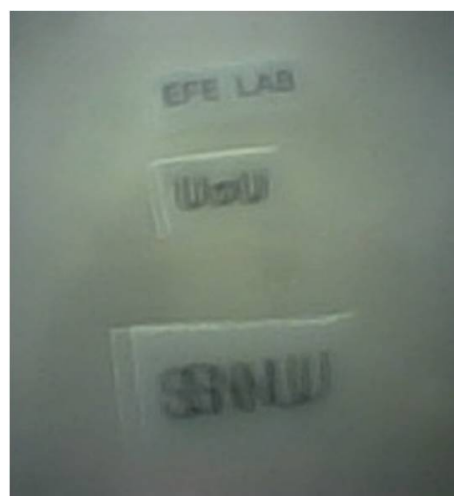

(b)

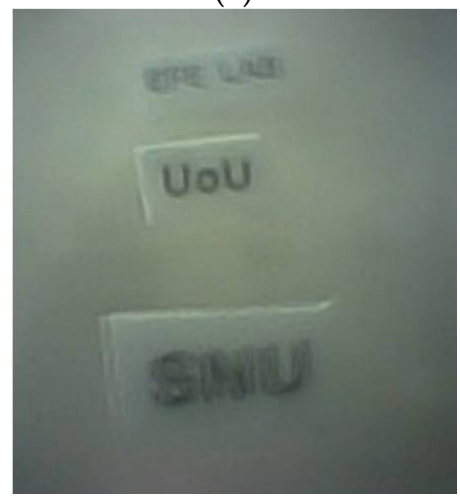

(c)

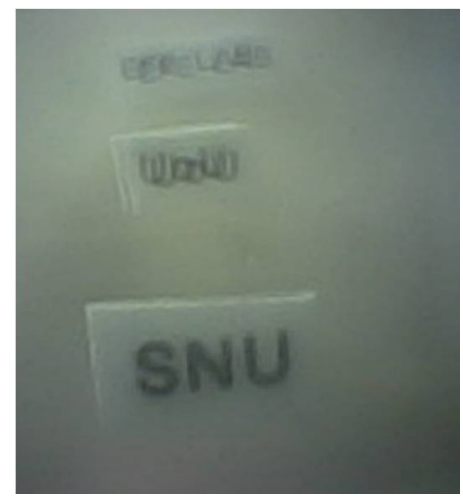

(d)

Figure 9. (a) The captured 18 subimages. (b) The reconstructed image focusing the "EFE LAB" apart $3 \mathrm{~cm}$ from the imaging system. (c) The reconstructed image focusing the "UoU" apart $2 \mathrm{~cm}$ from the imaging system. (d) The reconstructed image focusing the "SNU" apart $1 \mathrm{~cm}$ from the imaging system.

\section{Discussion \& Conclusions}

The imaging system mimicking an insect's compound eye was developed. Due to the light screening layer among the microlenses fabricated by our PLISL process and the adjustable jig, all of the 18 subimages were clearly focused. A simple change in the mask for the microlens patterning can increase the number of the subimages, which means that there is higher angular resolution and depth resolution in reconstructed images. Although the field-of-view of our imaging system is wider than other types of microlens array imaging systems and a conventional $35 \mathrm{~mm}$ camera with a compact lens system, it should be increased for recent emerging demands such as virtual reality and unmanned vehicles. Currently, we are researching a microlens array on hemispherical light sensors to meet these requirements. 
Supplementary Materials: The following are available online at http:/ /www.mdpi.com/1424-8220/18/7/2011/ s1.

Author Contributions: Conceptualization, J.-M.S. and K.K.; Methodology, M.S.; Software, M.S.; Validation, K.K.; Formal Analysis, M.S.; Investigation, M.S.; Resources, J.-M.S., D.C., and K.K.; Data Curation, M.S. and K.K.; Writing-Original Draft Preparation, M.S.; Writing-Review \& Editing, J.-M.S. and K.K.; Visualization, M.S.; Supervision, J.-M.S. and K.K.; Project Administration, J.-M.S., D.C. and K.K.; Funding Acquisition, J.-M.S., D.C. and K.K.

Funding: This research was funded by Defense Acquisition Program Administration, and by Agency for Defense Development (UD160027ID).

Acknowledgments: This research was supported by a grant to Bio-Mimetic Robot Research Center Funded by Defense Acquisition Program Administration, and by Agency for Defense Development (UD160027ID).

Conflicts of Interest: The authors declare no conflict of interest.

\section{References}

1. Ahuja, N.A.; Bose, N.K. Design of large field-of-view high-resolution miniaturized imaging system. EURASIP J. Adv. Signal Process. 2007, 2007, 1-10. [CrossRef]

2. Land, M.F. Compound eyes: Old and new optical mechanisms. Nature 1980, 287, 681-686. [CrossRef] [PubMed]

3. Horridge, G.A. The separation of visual axes in apposition compound eyes. Philos. Trans. R. Soc. B Biol. Sci. 1978, 285, 1-59. [CrossRef]

4. Snyder, A.W. Physics of vision in compound eyes. In Comparative Physiology and Evolution of Vision in Invertebrates: A: Invertebrate Photoreceptors; Autrum, H., Bennett, M.F., Diehn, B., Hamdorf, K., Heisenberg, M., Järvilehto, M., Kunze, P., Menzel, R., Miller, W.H., Snyder, A.W., et al., Eds.; Springer: Berlin/Heidelberg, Germnay, 1979; pp. 225-313.

5. McIntyre, P.; Caveney, S. Graded-index optics are matched to optical geometry in the superposition eyes of scarab beetles. Philos. Trans. R. Soc. B Biol. Sci. 1985, 311, 237-269. [CrossRef]

6. Rafael, N.; Nicolas, F. On image quality of microlens arrays in diurnal superposition eyes. Pure Appl. Opt. J. Eur. Opt. Soc. Part A 1998, 7, L69.

7. Park, H.S.; Hoskinson, R.; Abdollahi, H.; Stoeber, B. Compact near-eye display system using a superlensbased microlens array magnifier. In Proceedings of the 2015 28th IEEE International Conference on Micro Electro Mechanical Systems (MEMS), Estoril, Portugal, 18-22 January 2015; pp. 952-955.

8. Brückner, A.; Duparré, J.; Dannberg, P.; Bräuer, A.; Tünnermann, A. Artificial neural superposition eye. Opt. Express 2007, 15, 11922-11933. [CrossRef] [PubMed]

9. Stollberg, K.; Brückner, A.; Duparré, J.; Dannberg, P.; Bräuer, A.; Tünnermann, A. The gabor superlens as an alternative wafer-level camera approach inspired by superposition compound eyes of nocturnal insects. Opt. Express 2009, 17, 15747-15759. [CrossRef] [PubMed]

10. Floreano, D.; Pericet-Camara, R.; Viollet, S.; Ruffier, F.; Bruckner, A.; Leitel, R.; Buss, W.; Menouni, M.; Expert, F.; Juston, R.; et al. Miniature curved artificial compound eyes. Proc. Natl. Acad. Sci. USA 2013, 110, 9267-9272. [CrossRef] [PubMed]

11. Buschbeck, E.K. The compound lens eye of strepsiptera: Morphological development of larvae and pupae. Arthropod Struct. Dev. 2005, 34, 315-326. [CrossRef]

12. James, M.; Nandamuri, S.P.; Stahl, A.; Buschbeck, E.K. The unusual eyes of Xenos peckii (strepsiptera: Xenidae) have green- and uv-sensitive photoreceptors. J. Exp. Biol. 2016, 219, 3866-3874. [CrossRef] [PubMed]

13. Keum, D.; Jeon, D.S.; Kim, M.H.; Jeong, K.H. Artificial compound eye inspired by imaging principle of xenos peckii. In Proceedings of the 2015 Transducers-2015 18th International Conference on Solid-State Sensors, Actuators and Microsystems (TRANSDUCERS), Anchorage, AK, USA, 21-25 June 2015; pp. 403-406.

14. Borrelli, N.F.; Morse, D.L.; Bellman, R.H.; Morgan, W.L. Photolytic technique for producing microlenses in photosensitive glass. Appl. Opt. 1985, 24, 2520-2525. [CrossRef] [PubMed]

15. Tanida, J.; Kumagai, T.; Yamada, K.; Miyatake, S.; Ishida, K.; Morimoto, T.; Kondou, N.; Miyazaki, D.; Ichioka, Y. Thin observation module by bound optics (tombo): Concept and experimentalverification. Appl. Opt. 2001, 40, 1806-1813. [CrossRef] [PubMed]

16. Song, Y.M.; Xie, Y.; Malyarchuk, V.; Xiao, J.; Jung, I.; Choi, K.-J.; Liu, Z.; Park, H.; Lu, C.; Kim, R.-H.; et al. Digital cameras with designs inspired by the arthropod eye. Nature 2013, 497, 95-99. [CrossRef] [PubMed] 
17. Daly, D.; Stevens, R.F.; Hutley, M.C.; Davies, N. The manufacture of microlenses by melting photoresist. Meas. Sci. Technol. 1990, 1, 759. [CrossRef]

18. Norland Optical Adhesive 72. Available online: https://www.norlandprod.com/adhesives/noa\%2072.html (accessed on 23 March 2018).

19. Ikjoo, B.; Anthony, W.C.; Beomjoon, K. Transfer of thin au films to polydimethylsiloxane (pdms) with reliable bonding using (3-mercaptopropyl)trimethoxysilane (MPTMS) as a molecular adhesive. J. Micromech. Microeng. 2013, 23, 085016.

20. Axelevitch, A.; Apter, B.; Golan, G. Simulation and experimental investigation of optical transparency in gold island films. Opt. Express 2013, 21, 4126-4138. [CrossRef] [PubMed]

21. Sung Cheol, P.; Min Kyu, P.; Moon Gi, K. Super-resolution image reconstruction: A technical overview. IEEE Signal Process. Mag. 2003, 20, 21-36. [CrossRef]

22. Kim, J.; Jung, J.-H.; Jeong, Y.; Hong, K.; Lee, B. Real-time integral imaging system for light field microscopy. Opt. Express 2014, 22, 10210-10220. [CrossRef] [PubMed]

(C) 2018 by the authors. Licensee MDPI, Basel, Switzerland. This article is an open access article distributed under the terms and conditions of the Creative Commons Attribution (CC BY) license (http:// creativecommons.org/licenses/by/4.0/). 